\title{
L'inscription du tympan nord de San Miguel d'Estella
}

\section{Robert Favreau}

\section{Résumé}

Au tympan nord de San Miguel Estella en Navarre le Christ est représenté dans une mandorle quadrilobée sur laquelle est gravée inscription suivante NEC DEUS EST NEC HOMO PRESENS QUAM CERNIS IMAGO SET DEUS EST ET HOMO QUEM SAGRA FIGURAT IMAGO Cette formule si expressive n'est pas due à quelque crise iconoclaste qui aurait amené à réaffirmer solennellement l'utilité du culte des images. II s'agit de deux vers de Baudri de Bourgueil qui ont eu une fortune certaine puisqu'on les retrouve sous la même forme ou une forme très voisine abbaye de Saint-Denis la cathédrale de Ferrare sur une monstrance du début du XIIle siècle, et dans les textes qu'à la fin de ce même siècle Guillaume Durand cite au chapitre de son Rationale divinorum officiorum qui traite de la décoration des églises.

\section{Citer ce document / Cite this document :}

Favreau Robert. L'inscription du tympan nord de San Miguel d'Estella. In: Bibliothèque de l'école des chartes. 1975, tome 133, livraison 2. pp. 237-246;

doi : https://doi.org/10.3406/bec.1975.450029

https://www.persee.fr/doc/bec_0373-6237_1975_num_133_2_450029

Fichier pdf généré le 16/04/2018 


\section{L'INSCRIPTION DU TYMPAN NORD DE SAN MIGUEL D'ESTELLA \\ par}

Robert FAVREAU

Estella est une petite ville de Navarre, sise sur. l'Éga, affluent de la rive droite de l'Ėbre. Elle se développa à partir de la fin du $x^{e}$ siècle, soús l'impulsion de Sanche Ramirez, roi d'Aragon et de Navarre, et comprit plusieurs noyaux urbains qui se fondirent progressivement : quartiers de San Pedro de la Rua, sur le camino francès de Compostelle, puis de San Lazaro, hôpital que dota Garcia V le Restaurateur vers 1135-1150, enfin de San Miguel et de San Juan Bautista, à la fin du XII $^{\mathbf{e}}$ siècle. La plus connue des églises de ces différents quartiers est celle de San Miguel, grâce au très beau tympan historié de son portail nord ${ }^{1}$.

Ce tympan représente le Christ en majesté, dans une mandorle quadrilobée, entouré des symboles des quatre évangélistes et de deux personnages debout, un homme et une femme. Le Christ bénit de sa main droite et tient, dans sa main gauche, un médaillon sur lequel est gravé le chrisme entre l'alpha et l'omega. L'inscription suivante, commençant en bas et à gauche, fait le tour de la mandorle :

NEC DEUS EST NEC HOMO PRESENS QUAM CERNIS IMAGO

SET DEUS EST ET HOMO QUEM SACRA FIGURAT IMAGO.

1. René Crozet, L'art roman en Nasarre et en Aragon. Conditions historiques, dans Cahiers de civilisation médiévale, t. V (1962), p. 37, 51, et Recherches sur la sculpture romane en Navarre et en Aragon. V. Estella, ibid., t. VII (1964), p. 313-315 et pl. III; pour la situation et le développement de la ville, voir Vicente Bielza de Ory, Estella, estudio geografico de una pequeña ciudad navarra, dans Principe de Viana, t. XXIX (1968), p. 53-115 et planches.

BIBL. Éc. Chartes. 1975.2 
"Cette présente image que tu vois n'est ni Dieu ni homme, Mais il est Dieu et homme Celui que figure cette image sacrée. "

C'est à l'interprétation de cette formule expressive que voudrait s'attacher cette note.

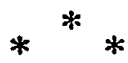

Le tympan historié de San Miguel d'Estella est bien connu, et il a été reproduit dans diverses publications ${ }^{1}$, mais l'inscription elle-même, plusieurs fois citée, n'a été commentée que par D. Tomás Biurrun y Sotil, qui s'est interrogé sur son sens : " Perogrullada? Puerilidad? Redundancia?" et a conclu qu'il s'agissait de réfuter les tendances iconoclastes ${ }^{2}$.

Certes, il était normal de se préoccuper en premier lieu de cette hypothèse. Très tôt dans l'Église chrétienne on s'était inquiété de cette difficulté qu'il y avait à représenter matériellement Dieu. Comment donc conférer " à la pierre et au bois un Nom incommunicable ", s'indignait déjà l'auteur du Livre de la Sagesse ${ }^{3}$ ? On s'employa à distinguer la représentation de ce qui était représenté, l'image de l'idole ${ }^{4}$, mais finalement il y eut crise profonde en Orient de 726 à 843, période au cours de laquelle, sauf une restauration passagère de 787 à 815 au temps de l'impératrice Irène, toutes les images furent prohibées à l'exception de la croix. Un des premiers actes de l'empereur iconoclaste Léon III fut de faire détruire l'image du Christ qui se trouvait à la

1. A. Kingsley Porter, Spanish Romanesque Sculpture, t. II, FlorenceParis, 1928, pl. 101 ; José Gudiol Ricart et Juan Antonio Gaya Nuño, Ars Hispaniae. Historia universal de l'arte hispanico. V. Arquitectura y escultura romanicas, Madrid, 1948, p. 171, fig. 289 ; R. Crozet, Recherches sur la sculpture romane en Navarre..., pl. III, fig. 9 ; Alain Sené, Quelques remarques sur les tympans romans à chrisme en Aragon et en Navarre, dans Mélanges offerts à René Crozet, Poitiers, t. I, 1966, pl. I, fig. 1 ; Navarre romane, La PierreQui-Vire, 1967, fig. 124 (Zodiaque).

2. El arte romanico en Navarra, Pxmplona, 1936, p. 205-214.

3. XIV, 21, et l'ensemble des chapitres XIII-XV. Voir aussi Exode, XX, 4-5; Deutéronome, V, 8-9; Isaie, XL, 18 ; Actes des apôtres, XVII, 29.

4. Sur le culte des images voir notamment Pierre Miquel, dans Dictionnaire de spiritualite, t. VII, deuxieme partie, Paris, 1971, col. 1503-1519 (avec bibliographie antérieure). 
Illustration non autorisée à la diffusion

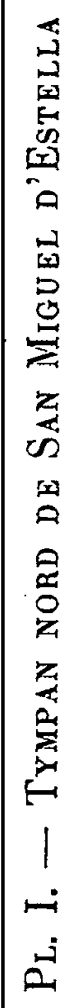


grande porte de bronze de l'entrée principale de son palais à Constantinople, et de la faire remplacer par une croix, avec l'inscription : "L'empereur ne peut admettre une image du Christ sans voix, sans souffle, et l'Écriture de son côté s'oppose à la figuration du Christ par sa (seule) nature humaine; voici pourquoi Léon et (son fils) le nouveau Constantin tracent sur la porte du palais le signe trois fois heureux de la croix, gloire des fidèles ${ }^{1}$. "Dans la première moitié du Ix siècle encore on trouvait en l'église Saint-Basile de Sinassos en Cappadoce une inscription auprès et autour d'une croix qui précisait : "Le Christ ainsi figuré ne subit aucun dommage, car on ne saurait le représenter par l'image ${ }^{2}$. "Toutefois, dès que prit fin la période iconoclaste, l'image du Christ fut replacée à la porte de bronze du palais impérial, et un office solennel célébra dès lors chaque année dans l'Église orthodoxe ce rétablissement du culte des images. En Occident il y eut crise aussi, à l'époque carolingienne, marquée surtout par le capitulaire sur les images, ou Libri carolini, composé vers 794, mais elle n'eut jamais l'acuité de celle qu'avait connue l'Empire byzantin, et il n'en est plus question passé la première moitié du $\mathrm{Ix}^{\mathbf{e}}$ siècle. Alors on peut se demander quels iconoclastes pouvait viser à la fin $\mathrm{du} \mathrm{xII}^{\mathrm{e}}$ siècle l'inscription de San Miguel d'Estella, notamment en Occident où on avait toujours souligné la fonction pédagogique de l'image, fonction rappelée alors par des auteurs aussi connus que Suger ${ }^{3}$ ou Honorius dit d'Autun ${ }^{4}$.

Or précisément en la basilique de Saint-Denis au temps de l'abbé Suger avait été établi un retable en pierre, sur lequel était représenté le Christ assis, dans un quatre-

1. André Grabar, L'iconoclasme byzantin. Dossier archéologique, Paris, 1957, p. 133 (Collège de France. Fondation Schlumberger pour les études byzantines).

2. Nicole Thierry, Les églises rupestres, dans Arts de Cappadoce, GenèvePeris-Munich, 1971, p. 162 a, et p. 137, fig. 72 .

3. Mens hebes ad verum per materialia surgit "(Suger, Liber de rebus in administratione sua gestis, éd. A. Lecoy de La Marche, Eusres complètes de Suger, Paris, Société de l'histoire de France; 1867, p. 189).

4. "Ob tres autem causas fit pictura : primo, quia est laicorum litteratura; secundo, ut domus tali decore ornetur; tertio, ut priorum vita in memoriam revocetur " (Gemma animae sive de divinis officiis..., dans Patr. lat., t. CLXXI, col. 586). 
feuilles, entouré des quatre animaux symboliques. Sur les bords du quatre-feuilles on lisait, gravés en lettres capitales, les deux vers suivants ${ }^{1}$ :

HIC DEUS EST ET HOMO QUEM PRESENS SIGNAT YMAGO

ERgo Rogabit homo QUEM SCULTA FIGURAT YMAGo.

Ce texte est fort proche de celui d'Estella et accompagne un groupe sculpté identique à celui d'Estella. Une inscription qui figurait sur une monstrance en métal doré du début $d u$ xrrre siècle, auprès d'une figure du Christ, se rapprochait plus encore des vers inscrits à Estella ${ }^{2}$ :

HIC DEUS EST NEG HOMO QUEM PRESENS SIGNAT IMAGO

SED DEUS EST ET HOMO QUEM SCULPTA FIGURAT IMAGO.

Enfin un autre texte jadis gravé au tympan de la Porta dei $M e s i$ à la cathédrale de Ferrare reproduisait presque exactement celui de San Miguel $d^{j}$ Estella. En effet, auprès du Christ écrasant l'aspic et le basilic et bénissant de sa main droite, on lisait ${ }^{3}$ :

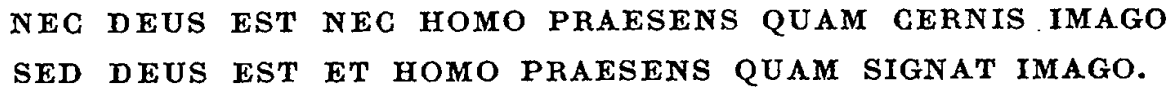

Il y a tant de concordance entre ces différents textes qu'il faut penser à une inspiration commune. Or cette inspiration existe, puisque les deux vers léonins inscrits à San Miguel d'Estella se trouvent dans les œưrres de Baudri, abbé de Bourgueil, mort en $1130^{4}$. Il s'agit d'un texte qui

1. F. de Guilhermy, Inscriptions de la France du Ve au $X V I I I^{\mathrm{e}}$ siècle. Ancien diocèse de Paris, Paris, t. II, 1875, p. 123-124 (Documents inédits sur l'histoire de France). Il s'agit d'hexamètres léonins, avec une erreur de quantité pour ergo.

2. Ibid., p. 124. Sans doute faut-il corriger le hic initial en nec.

3. Arthur Kingsley Porter, Lombard Architecture. Volume II : Monuments. Abbazia di Albino-Millan, New Haven-Londres-Oxford, 1916, p. 416 et n. 47, d'après une description de Borsetti publiée en 1735 : "In ejusdem vero semicirculo Redemptoris effigies, aspidem ac basiliscum concultantis, ac dexteram in benedicentis modum protendentis prominebat, his additis carminibus...

4. Les cusres poétiques de Baudri de Bourgueil (1046-1130), édition critique 
eut une certaine fortune puisqu'on le rencontre dans trois manuscrits sans nom d'auteur (Vatican, Paris, Vienne) et au cours d'un traité de visitatione infirmorum qui est probablement de Baudri. Il avait été attribué, dans la Patrologie latine, à Hildebert de Lavardin ${ }^{1}$, mais cette attribution avait été corrigée par Hauréau ${ }^{2}$. Les variantes sous lesquelles le présentent les différents manuscrits ne concernent que la fin des vers, et correspondent chaque fois à une formule facile à retenir grâce à sa composition et grâce à la métrique :

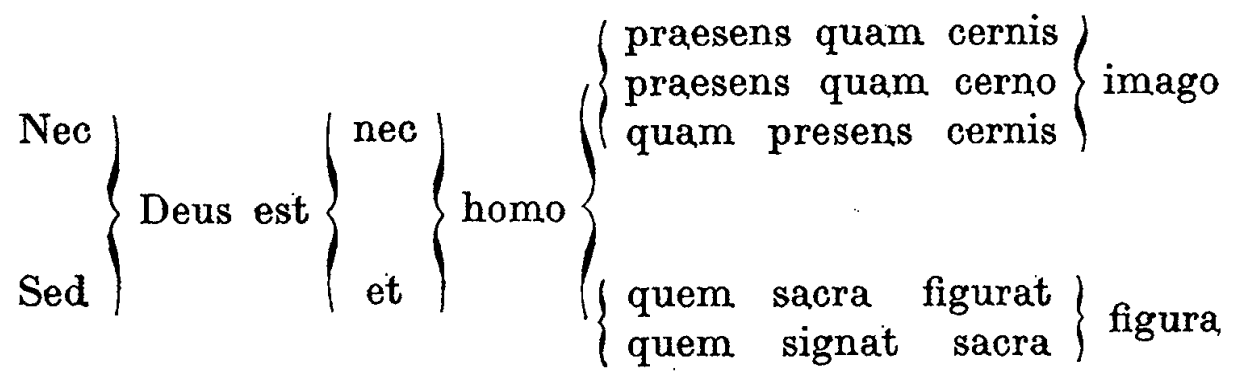

Il s'agit donc d'un texte fort répandu, qui a été " aménagé » au fur et à mesure des transmissions.

Un des manuscrits qui ont conservé ces vers les présente comme dirigés " contra Judeos et ereticos et saracenos qui dicunt nos adorare idolas ${ }^{3}$ ». On ne peut écarter toute intention apologétique, car on sait que les musulmans, alors solidement implantés en Espagne, se refusaient à reproduire la figure humaine, et plus de la moitié des tympans navarrais et aragonais ne sont ornés que du chrisme. On ne peut d'ailleurs que s'interroger sur l'exceptionnelle fréquence de l'emploi du chrisme de part et d'autre des Pyré-

publiée d'après le manuscrit du Vatican par Phyllis Abrahams, Faris, 1926, p. 182, no CLXXXVIII, Circa crucifixum.

1. Patr. lat., t. CLXXI, col. 1283, Inscriptionum christianarum libellus, XXXII, Ubi Christus pingitur imago, et col. 1426, Carmina miscellanea, LXXXVI, Ad Christi crucifixi imaginem.

2. Barthélemy Hauréau, Notices des manuscrits latins 583,657, 1249, 2945, $2950,3145,3146,3437,3473,3482,3495,3498,3652,3702,3730$ de la Bibliothèque nationale, dans Notices et extraits des manuscrits de la Bibliothèque nationale et autres bibliothèques publiés par l'Académie des inscriptions et belles-

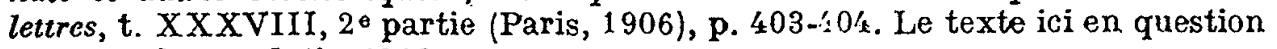
est dans le ms. latin 1249.

3. Bibl. Vaticane, ms. Reg. 1578, fol. 46. 
nées ${ }^{1}$, et à Estella même, seul au centre du tympan de San Lazaro $^{2}$ et du portail de San Pedro de la Rua. Mais la formule bien frappée qui figure à San Miguel d'Estella correspond aussi certainement à cette difficulté générale qu'a toujours ressentie l'artiste chrétien pour représenter Dieu, et ce indépendamment de toute tendance iconoclaste ${ }^{3}$. Peut-être ne faut-il pas chercher plus loin la justification de cette inscription.

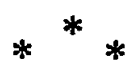

L'emploi en Navarre de ces deux vers est intéressant à retenir pour la diffusion de l'œuvre de Baudri de Bour-

1. La carte ci-contre est établie à partir des chiffres donnés, pour la France, par Paul Mesplé, Les chrismes du département du Gers. Observation sur l'évolution et le groupement des chrismes au nord et au sud des Pyrénées, dans Mémoires de la Société archéologique du Midi de la France, t. XXXV (1970), p. 71-88; le chiffre de 32 fourni par cette étude pour les Pyrénées-Atlantiques a été corrigé en 39 a partir de la confrontation de la liste de ces chrismes avec celle établie par Constant Lacoste, Les chrismes des églises béarnaises, dans Bulletin de la Société des sciences, lettres et arts de $P$ au, $4^{\circ}$ série, $t$. V, 1970, p. 53-71. Les chris mes sont surtout abondants au centre de la région pyrénéenne, puisqu'il n'y en a aucun dans les Pyrénées-Orientales, tandis que dans les Pyrénées-Atlantiques la quasi-totalité (38) se trouvent en Béarn, le dernier se situant en Haute-Soule. Pour l'Espagne L. Torrès Balbas, La escultura románica aragonesa y el crismón de los tímpanos de las íglesias de la región pirenáica, dans Archivo Español de Arte y Arqueología, t. II, 1926, p. 287-290, en déno mbrait 38 de Lérida à Compostelle, avec une densité maximale en Aragon et en Navarre; les chiffres de $2 /$ pour l'Aragon et de 15 pour la Navarre donnés par A. Sené (art. cité, p. 367) ne peuvent être considérés comme un état exhaustif (Léon Pressouyre, Chronique, dans Bulletin monumental, t. CXXV (1967), p. 193-194, et P. Mesplé, art. cité, p. 86).

2. José Gudiol, San Salvador de Leyre y el primer romanico en Navarra, dans Principe de Viana, t. V (1944), p. 266 et fig. 65 hors texte; Luis Vazquez de Parga, José Maria Lacarra, Juan Uría Ríu, Las peregrinaciones a Santiago de Compostella, t. III, Madrid, 194:9, pl. LX. Ce tympan est aujourd'hui conservé dans les collections archéologiques de Pampelune.

3. James Stuart Morgan, Le temps et l'intemporel dans le décor de deux églises romanes : facteurs de coordination entre la mentalité religieuse romane et les ousres sculptées et peintes à Saint-Paul-lès-Dax et à Saint-Chef en Dauphiné, dans Mélanges offerts à René Crozet, t. I, p. 542.543. Le tympan ro man de l'église auvergnate de Champeix offre un bon exemple de ce malaise de l'artiste devant une représentation anthropomorphique de Dieu : la 'Trinité y est, en effet, figurée par la Main divine, l'Agneau crucifère et la Colombe, avec l'inscription :

TRES TRINUH SIGHENT ; POHAEX, PEGCUS ATQUE GOJUMRA. 
Illustration non autorisée à la diffusion 
gueil ${ }^{1}$. Il pose encore le problème des modèles suivis ici et là lorsqu'on désirait graver quelque inscription. L'épigramme a constitué, dès les Romains, un véritable "genre " littéraire. Paulin de Périgueux et Venance Fortunat au vie siècle, Alcuin, Théodulphe, Raban Maur à l'époque carolingienne ${ }^{2}$, continuèrent une tradition ancienne qu'aux $\mathrm{XI}^{\mathrm{e}}$ et $\mathrm{XII}^{\mathrm{e}}$ siècles poursuivirent, entre autres, Foulcoie de Beauvais ${ }^{3}$, Hildebert de Lavardin et Baudri de Bourgueil: Ainsi ces deux derniers composèrent, chacun de leur côté, une épitaphe pour Bérenger de Tours ( $\dagger$ 1088); celle d'Hildebert ne comportait pas moins de cinquante-deux vers, et on sait que les deux premiers furent effectivement gravés sur la tombe de Bérenger ${ }^{4}$. Mais pour apprécier exactement les emprunts qui furent faits à ces différents auteurs il faudrait disposer d'un recueil aussi complet que possible des inscriptions médiévales, avec l'accompagnement de tables développées. Un tel recueil est en cours, pour la France, au Centre d'études supérieures de civilisation médiévale de Poitiers, et les recoupements que permettent déjà les fichiers s'avèrent fort fructueux $\mathbf{x}^{5}$ : Mais pour bien saisir quels étaient les auteurs qui formaient la base de la culture des épigraphistes aux $\mathbf{x I}^{\mathbf{e}}, \mathbf{x I I}^{\mathbf{e}}, \mathbf{x I I I}^{\mathbf{e}}$ siècles, il faudrait une recension étendue à tout l'Occident, à la manière du Corpus inscriptionum latinarum.

1. L’abbé Henri Pasquier, Un poète latin du XIo siècle. Baudri abbé de Bourgueil, archevêque de Dol (1046-1130), Paris-Angers, 1878, p. 269, note que les vers de Baudri passèrent les Alpes et charmèrent les loisirs de l'évêque d'Ostie.

2. Voir notamment $M$. G. H., Poetae latini aepi carolini, éd. E. Dümmler, t. I et $\mathrm{II}_{\mathrm{b}}$, Berlin, 1880-1 8.84.

3. Henri Omont, Epitâphes métriques en l'honineur de différents personnages du XI० siècle, composées par Foulcoie de Beausais, archidiacre de Meaux, dans Mélanges Julien Havet, Paris, 1895, p. 211-236.

4. B. Hauréau, Noticé sur les mélanges poétiques d'Hildebert de Lavardin, dans Notices et extraits des manuscrits de la Bibliothèque nationale et autres bibliothèques publiés par l'Institut national de France, t. XXVIII, $2^{\theta}$ partie (Paris, 1888), p. 308-311.

5. Robert Favreau, L'épigraphie médiévale, dans Cahiers de civilisation médiésale, t. XII (1969), p. 393-398. Le fichier bibliographique général recense actuellement environ dix mille inscriptions; 1'examen sur place, avec photographie ou estampage, est réalisé pour les régions de Poitou-Charentes et de Limousin, soit sept départements; un premier fascicule a été publié en $197 \%$, et un second le sera à la fin de 1975. La présente note est issue d'un enseignement d'épigraphie médiévale qui, lui, ne s'arrête évidemment pas aux frontières de la France. 
De la culture de son temps l'évêque de Mende Guillaume Durand a été, à la fin $d u$ xıı ${ }^{\mathbf{e}}$ siècle, un des meilleurs révélateurs qui soient, et quiconque s'intéresse à l'épigraphie trouve toujours profit à le consulter. Or Durand, au chapitre III du livre I de son Rationale divinorum officiorum, traite des peintures, tapisseries et ornements des églises, et, après avoir cité une lettre de Grégoire le Grand à l'évêque de Marseille Serenus ${ }^{1}$ — "... aliud est picturam adorare, aliud per picturae historiam quid sit adorandum addiscere..." - il donne trois exemples de vers qui se rapportent au culte des images tel qu'il faut l'entendre. Le deuxième de ces exemples n'est autre que le distique de Baudri de Bourgueil inscrit à San Miguel d'Estella. Le troisième est de proche facture :

Nam Deus est quod imago docet; sed non Deus ipsa.

Hanc videas, sed mente colas quod noscis in ipsa ${ }^{2}$.

Il se trouve que ce dernier distique figure au-dessus du Christ dans une mosaĩque de la chapelle Saint-Clément en la basilique Saint-Marc à Venise ${ }^{3}$, et le Bâlois Thomas Platter vit, en 1599, exactement le même texte en l'église aujourd'hui disparue de Saint-Didier de Poitiers ${ }^{4}$. Citer d'autres inscriptions de même genre ${ }^{5}$ entraînerait à une recherche qui

1. Patr. lat., t. LXXVII, col. 1128.

2. Guillaume Durand, Rationale divinorum officiorum, Lyon, 1559, fol. $12 \mathrm{v}^{\circ}$.

3. Camillo Boïto, La basilique de saint Marc à Venise, trad. A. Cruvellié, t. III, Venise, 1890 , p. 855 . Cette inscription m'a été aimablement signalée par M. Henri Renou, photothécaire au C. E. S. C. M. ; je l'en remercie vivement.

4. En outre j'ai encore vu l'église Saint-Didier oủ ces vers étaient écrits sous la grande croix :

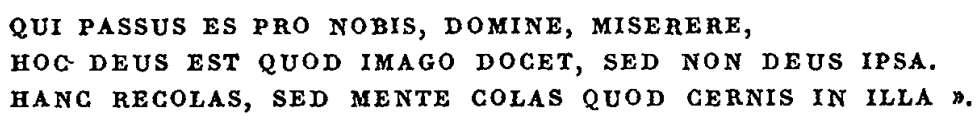

(Gaston Dez; Un visiteur inconnu du Poitou et de Poitiers en 1599; le Bâlois Thomas Platter, dans Bulletin de la Société des antiquaires de l'Ouest, $4^{\circ}$ série, t. XI, 1971-1972, p. 573).

5. Une des plus intéressantes est celle qui figure sur le devant d'autel de l'église Saint-Victor à Xanten, qui est daté de 1129. Elle est inscrite sur la mandorle qui entoure le Christ en Majesté, entre les évangélistes et les prophètes :

RES ET IMAGO DUAS FERT ISTA NOTATQUE FIGURAS, EFFIGITUS HOMO, DEUS EST SIGNATUS IN AURO.

(Franz-Xaver Kraus, Die christlichen Inschriften der Rheinlande, t. II, Fribourg-en-B. et Leipzig, 1894, n 650, p. 297-298). 
dépasserait singulièrement le cadre de cette présente note. Celle-ci n'a d'autre ambition, tout en éclairant l'inscription du beau tympan de San Miguel d'Estella, que d'attirer l'attention sur la nécessité de constituer un recueil général des inscriptions médiévales, car c'est ainsi seulement qu'on arrivera à mieux comprendre le sens de tous ces textes qu'on peut encore lire aujourd'hui un peu partout dans les églises et leurs trésors, et par là qu'on pénétrera mieux la culture et la mentalité médiévales.

Robert Favreau. 